\title{
THE OPERATIONAL ENVIRONMENT IN THE AGE OF ARTIFICIAL INTELLIGENCE. POSSIBLE SCENARIOS.
}

\author{
Laviniu BOJOR \\ "Nicolae Bălcescu" Land Forces Academy, Sibiu, Romania \\ laviniu.bojor@gmail.com
}

\begin{abstract}
The permanent tendency to invest huge budgets of money in research and development in order to create military capabilities that will allow hitting a large number of targets in a short time and in an extended battlespace continues to be the main direction for neutralizing a possible opponent in the military conflicts. Due to current scientific and technological advances, the focus nowadays is on using artificial intelligence (AI) with the purpose of dominating the operational environment in the theatres of operation (TO). The present paper aims to identify how AI will shape the operational environment of future military conflicts.
\end{abstract}

KEYWORDS: artificial intelligence, Narrow AI, Strong AI, Singularity, Skynet, Immortality

\section{Introduction}

Drones, robots, learning machines and unmanned vehicles are the elements by which military scholars characterize future armed conflicts. No one is considering an operating environment lacking state-of-the-art technology equipment and this rise of Artificial Intelligence (AI) is based on performance well above the average human level that machines have been able to achieve in an extremely short time. The ubiquity of sensors and smart devices in military equipment makes AI listed as the no. 1 "strategic technology" (Duan, Edwardsb \& Dwivedic, 2019). The opportunities offered by the use of technology but also the impossibility to fully understand the functioning of these "black boxes" or even the fear that machines can turn against humanity leads to divergent opinions regarding the future of AI. Feelings "both anxiety and ambition" (Keating \& Nourbakhsh, 2018, p. 29) are felt when it comes to the future human-machine collaboration, and this article aims to identify the possible scenarios of the operational environments marked by Ai depending on the development stages achieved.

\section{From Narrow AI to Singularity}

Artificial intelligence, this "the ability of computers to mimic human thought" (Panchal, 2018) can be classified by the stages towards which AI evolved: Narrow/Weak AI and Strong AI. Narrow $A I$ is the artificial intelligence of the present, being characterized by the ability to perform specific tasks: voice and image recognition, virtual assistance (Amazon Alexa, Apple Siri, Google Assistant), purchase suggestions, sales predictions, weather forecasts, playing games 
(chess, Go, Jeopardy, poker), autonomous cars, basic translations or text-to-speech. Narrow IA is characterized as extremely fast and clever in the domain it was created for, but it is limited to performing pre-defined sets of functions, without having emotions, beliefs, sensitivity or consciousness. Narrow $\mathrm{AI}$ is able to beat the world champion at GO, considered one of the most creative games in the world, but the same algorithm is extremely limited in performing other tasks. Strictly within the military context, loading drones/robots with the learning/perfecting algorithm of the "war" game would result in the immediate neutralization of a human adversary. We must also find the opponent who is willing to fight a robot.

In the next stage we could have access to human-level AI or Strong AI, the one "that can understand and reason its environment as a human would" (Dickson, 2017). This would be capable of selflearning, making connections, connecting itself to the architecture of the Internet and amplifying its capabilities by accessing Big Brother and IoT data, collecting the knowledge learned by other algorithms, being innovative, creative and confident in making decisions under pressure. "Over time these intelligences would be able to take over every role performed by human" (Heath, 2018). The exponential increase of AI power will combine all developed skills in one entity, which, although not present yet, has been named singularity technology (Figure 1). A consciousness machine smarter than humans. Most analysts in AI field agree with it, but it is very difficult to estimate the date when it will appear, 2045 (Reedy, 2017), 2040 (Galeon, 2018) or even earlier, in 2030 (Ganapati, 2010). Probably, their estimations must be updated according to the information on the changes in technology from Google quantum field, "replacing traditional bits the 'ones' and 'zeros' used in digital communications - with quantum bits, or qubits" (Cuthbertson, 2019).

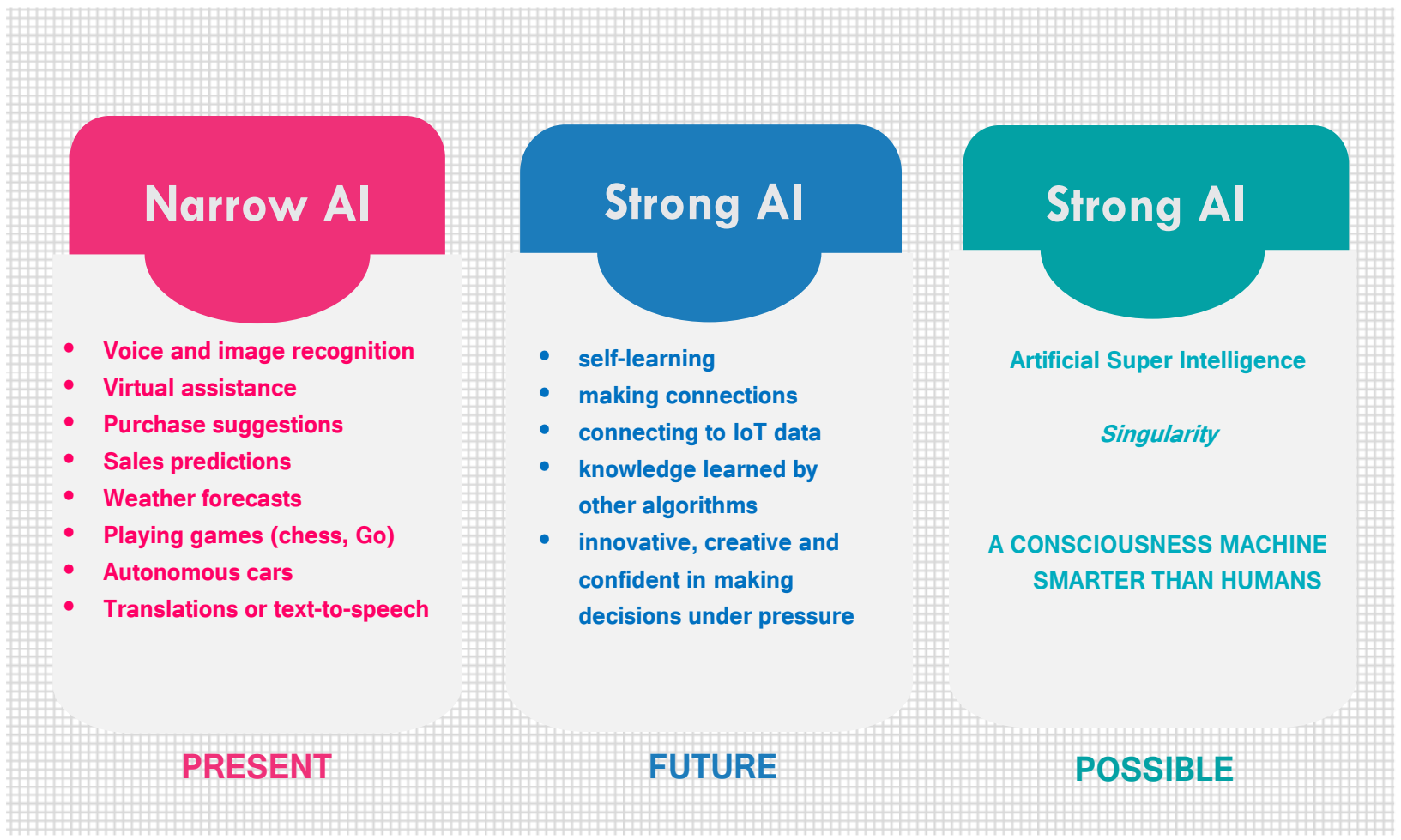

Figure no. 1: AI evolutionary stages 
In this respect, we can identify 3 main directions of transformation of the operational environment $(\mathrm{OE})$ in accordance with $\mathrm{AI}$ evolution.

\section{Skynet Loading}

My military background taught me to consider the most dangerous course of action (MDCOA) a priority. This would mean an operational environment similar to a SF movie screenplay where the equipment specific to the Internet of Things will provide data and information to the algorithms of some learning machines which will pass from "Strong" AI (Artificial General intelligence) to ASI (Artificial Super Intelligence) and that super power, singularity technology, would turn against humanity. Probably, I am still marked by the Terminator movie, which I saw when I was a child, and by the many mistakes of some remotely guided missiles. If AI get to dominate us, I suppose the problem will no longer be if it can, but if it wants to wipe us out. It will study all types of conflict that have existed in time by accessing online military and civilian libraries. It will connect wirelessly to all military networks and control the entire nuclear arsenal, not just that of NATO, Russia or China. It will take over all Unmanned Vehicles (like American aerial Pegasus and Russian Poseidon underwater drones) and it will guide them to strike using information from the Earth's artificial satellite networks (GPS, GLONASS, GALILEO). Where it cannot use unmanned aircraft, it will send swarm squadrons of hummingbirds size drones, military or civilian, belonging to some states or owned by private companies. It will take out of action all the ground fighting means that it cannot control (armored, airplanes) by destroying the sensors that make their proper functioning possible. It will activate its Boston Dynamics robots and other DARPA innovations to protect itself against any attempts of humans to "pull the cable out of the socket". This Skynet scenario shows us that it is not a question of whether the ASI can exterminate us but whether it wants to. One of the reasons would be that AI will reach a level where man becomes the useless 'village idiot' that machines have to take care of. We do not believe that there is anyone who can say that we will accept to be slaves of robots but the machine learning power will reach a level at which the human will remain well below their intelligence level. Machines will program their own learning algorithms, they will use a coded language that we cannot break, they will develop their own army of bots, chatbots and robots to meet their needs and goals. And when their AI needs are not aligned with human needs, village idiots will become an obstacle to the progress of the new cyber society. The asymmetry will be more than obvious. The man will fight horseback, with Kalasnikov or other sensor-free weapons, he will finds his way on the map with a compass without GPS, he will not have access to IMINT, SIGINT or GEOINT, nor will he have the opportunity to communicate with other comrades, because radio or satellite communications will be jammed for people.

\section{Immortality}

If the first scenario would lead to the extermination of the human race, the second scenario is a positive one, even utopian, and it can bring us immortality. We still find AI at a higher level than man; however, the power attained by singularity will not turn against us but will help us to solve many, if not all, the problems of the present (Figure no. 2):

- Identifying alternative, nonpolluting sources of energy to stop $\mathrm{CO} 2$ emissions and global warming;

- Identifying and remedying diseases that destroy society, such as cancer;

- Eliminating food insecurity and hunger by accessing new artificial food sources, which will be distributed evenly worldwide; 
- Eliminating urban traffic by improving the transport system (local, regional and global);

- Slowing down aging or even achieving immortality) (Urban, 2015) with the technological advancement in medicine, genetics, neuroscience, biotechnology, nanotechnology and other cognitive sciences.

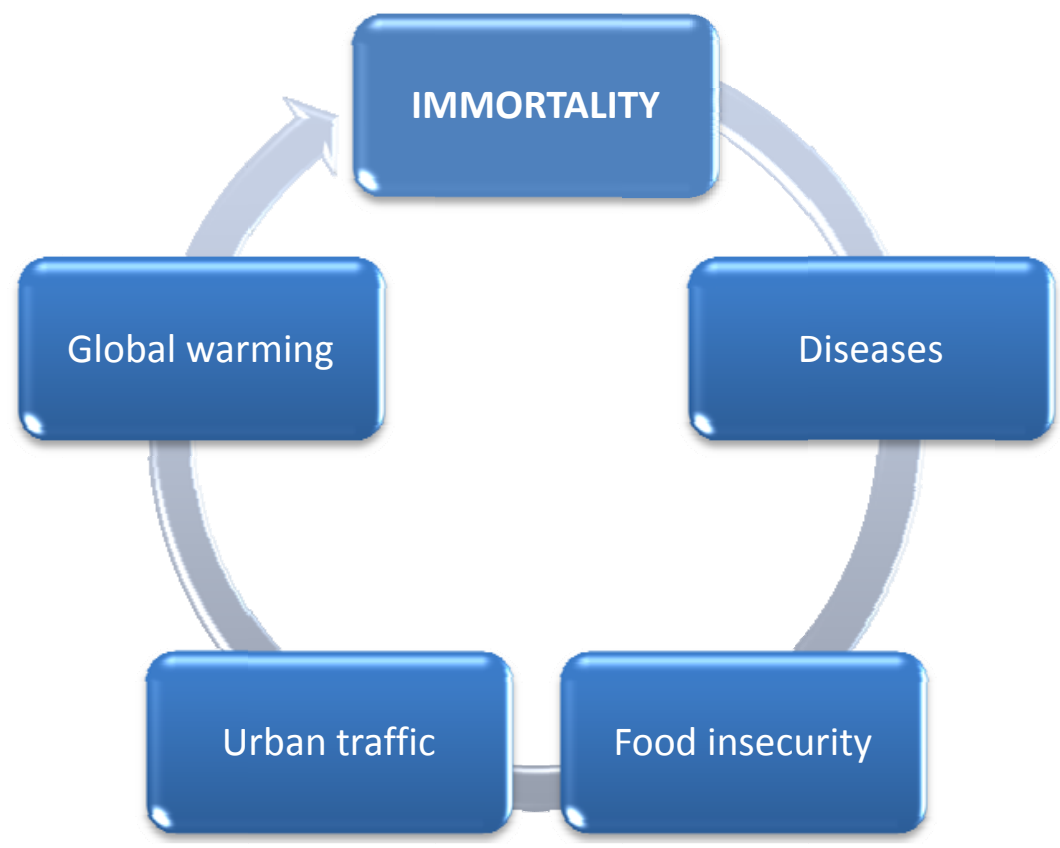

Figure no. 2: Singularity can solve world's biggest problems

In this hypothetical scenario, conflicts will disappear because there will be no political divergences, struggles for power or resources, or other challenges that will require the opening of new theaters of military operations.

Singularity will not accept being manipulated for the purpose of pursuing particular interests (even if they come from the state or the non-state that created it) and will lay the foundations of a new world that offers stability and security for everyone, regardless of race, nationality or gender. A utopian world without terrorism and conflicts, without criminals or vandalism, so without the need to maintain large armies. A transparent world, without vices and without secrets to which ASI does not have access. A perfect man, stripped of hatred, pride, greed, envy or fear. A perfect man in a perfect world. And we, the military, will have to look for new jobs.

\section{Narrow AI World}

The third scenario is the one in which the operational environment of the armed conflicts will continue to be addicted to smart devices but the AI level will not exceed the human one and the decisionmaking process will be fully controlled by the people. Soldiers equipped with exoskeletons and military leaders connected to information networks capable of providing real-time images and videos will mark the future of the OE, one that will surely take place in the urban environment and will be marked by the irregularity that allows the opponent to hide his identity behind the human shield made up of children, women and the elderly. It is the scenario closest to the present and the one that will customize the theaters of military operations in the near future, until the emergence and development of a God-like ASI. 


\section{Conclusions}

The decision to set limits and regulations to stop the AI advancement towards Singularity does not belong to military theorists and practitioners. No one knows for sure whether ASI will be good or bad but the decision to reach this level is not ours. It will remain a political tool of high-tech states, such as the USA, which invest in agencies such as DARPA, in order to maintain or gain a military advantage over potential aggressors such as Russia or China. But even if the great powers unanimously decide to put some limits to the attempt of going over the superintelligence level, especially by limiting the allotted budgets, the technological effort in the civilian environment cannot be stopped. Non-state actors, such as Google, Apple, Facebook or Tesla, will continue to invest in $\mathrm{AI}$ in order to have an economic advantage in the ongoing global competition that man has created in his brief existence on Earth. It is like the global warming: everyone knows that the planet is suffocating but no one slows down the engine. The best example is Elon Musk who claims that $\mathrm{AI}$ is more dangerous than nuclear missiles (Clifford, 2018) but he invests in his new company - Neuralink which aims to accelerate this process by transferring human consciousness to digital devices using the brain-computer interface (Musk, 2019). By the time we see if ASI is a threat or a miracle we will have to prepare for the military confrontations of the near future, marked by Narrow AI. This means avoiding vulnerabilities and taking advantage of the opportunities generated by the ubiquity of smart devices, sensors, applications, algorithms or learning machines. In this sense, military personnel must know all the characteristics of high-tech military equipment and use them efficiently, both during intelligence collection, analysis, filtering and dissemination, but especially during the military decision-making process.

\section{REFERENCES}

Clifford, C. (2018). Elon Musk: 'Mark my words - A.I. is far more dangerous than nukes'. CNBC International, available at: https://www.cnbc.com/2018/03/13/elon-musk-atsxsw-a-i-is-more-dangerous-than-nuclear-weapons.html, accessed on 05 October 2019.

Cuthbertson, A. (2019). Google 'achieves quantum supremacy'. INDEPENDENT, available at: https://www.independent.co.uk/life-style/gadgets-and-tech/news/googlequantum-supremacy-computers-nasa-a9116371.html, accessed on 05 October 2019.

Dickson, B. (2017). What is Narrow, General and Super Artificial Intelligence. TechTalks, available at: https://bdtechtalks.com/2017/05/12/what-is-narrow-general-and-super-artificialintelligence/, accessed on 08 October 2019.

Duan,Y., Edwardsb, J. S., \& Dwivedic, Y. K. (2019). Artificial intelligence for decision making in the era of Big Data - evolution, challenges and research agenda. International Journal of Information Management, Vol. 48. https:// doi.org/ 10.1016/ j.ijinfomgt.2019.01.021.

Galeon, D. (2018). Separating Science Fact from Science Hype: How Far off Is the Singularity? Most experts are confident it will happen sooner rather than later. Futurism, available at: https://futurism.com/separating-science-fact-science-hype-how-far-offsingularity, accessed on 10 October 2019.

Ganapati, P. (2010). Reverse-Engineering of Human Brain Likely by 2030, Expert Predicts. WERED, available at: https:// www.wired.com/2010/08/reverse-engineering-brainkurzweil/, accessed on10 October 2019. 
Heath, N. (2018). What is artificial general intelligence? A ZDNET, available at: https://www.zdnet.com/article/what-is-artificial-general-intelligence/, accessed on 12 October 2019.

Keating, J., \& Nourbakhsh, I. (2018). Teaching Artifcial Intelligence and Humanity. Magazine Communications of the ACM, Vol. 61, Issue 2, 29. DOI:10.1145/3104986.

Musk, E. (2019). Neuralink presentation, available at: https://www.youtube.com/ watch? $\mathrm{v}=\mathrm{dXbShyPQrhM}$, accessed on 18 October 2019.

Panchal, S. (2018). Types of Artificial Intelligence and examples. Medium, available at: https://medium.com/predict/types-of-artificial-intelligence-and-examples-4f586489c5de, accessed on 22 October 2019.

Reedy, C. (2017). Kurzweil Claims that the Singularity Will Happen by 2045. Get ready for humanity 2.0. Futurism, available at: https://futurism.com/kurzweil-claims-that-thesingularity-will-happen-by-2045, accessed on 25 October 2019.

Urban, T. (2015). The AI Revolution: Our Immortality or Extinction, available at: https://waitbutwhy.com/2015/01/artificial-intelligence-revolution-2.html, accessed on 30 October 2019. 